Rev. Inst. Flor. v. 26 n. 2 p. 169-181 dez. 2014

http://dx.doi.org/10.4322/rif.2014.013

ISSN impresso 0103-2674/on-line 2178-5031

\title{
COMPARAÇÃO DE ASPECTOS FENOLÓGICOS E DE FRUGIVORIA ENTRE A PALMEIRA NATIVA Euterpe edulis Mart. E A PALMEIRA EXÓTICA Phoenix roebelenii O'Brien (ARECACEAE) NO SUDESTE DO BRASIL ${ }^{1}$
}

\author{
COMPARATIVE FRUIT PHENOLOGY AND FRUGIVORY BETWEEN THE \\ NATIVE PALM Euterpe edulis Mart. AND THE EXOTIC PALM Phoenix roebelenii O'Brien \\ (ARECACEAE) IN SOUTHEASTERN BRAZIL
}

\author{
Taís OMOTE²; Alexsander Zamorano ANTUNES², ${ }^{2}$ Ciro Koiti MATSUKUMA²
}

\begin{abstract}
RESUMO - A dispersão de sementes de vegetais exóticos por animais nativos é um dos fatores associados a invasões biológicas. Visando colaborar para maior compreensão sobre o estabelecimento dessa relação, os objetivos do presente trabalho foram comparar os padrões fenológicos de frutificação e caracterizar a assembleia de aves visitantes de duas palmeiras, a nativa Euterpe edulis e a exótica Phoenix roebelenii no Parque Estadual Alberto Löfgren. Phoenix roebelenii é originária do sudeste da Ásia, frequentemente utilizada no paisagismo urbano, e apresenta-se disseminada no sub-bosque florestal do Parque Estadual Alberto Löfgren. Contudo, ela não é considerada uma espécie invasora em território brasileiro. Os padrões fenológicos de 30 indivíduos adultos de cada espécie de palmeira foram acompanhados mensalmente entre abril de 2012 e março de 2014. A caracterização das assembleias de aves visitantes foi obtida por meio de observação focal. As palmeiras diferiram em seus padrões fenológicos apresentando sobreposição parcial na fenofase frutos maduros, mas não em seus picos de frutificação. Considerando-se apenas os dispersores em potencial de ambas as espécies, observou-se que aves distintas foram responsáveis pelas maiores taxas de consumo de frutos obtidas para cada uma das palmeiras. Os dados do presente trabalho sugerem que a palmeira exótica se tornou importante fonte de alimento para aves nativas nessa unidade de conservação, e que em decorrência dessa interação ocorre a dispersão de sementes, a qual provavelmente contribui para o recrutamento bem-sucedido dessa planta no local, o que justificaria o desenvolvimento de pesquisas sobre o impacto do seu estabelecimento sobre as espécies vegetais nativas.
\end{abstract}

Palavras-chave: Aves; invasão biológica; Mata Atlântica.

\begin{abstract}
Seed dispersal of exotic plants by native animals is one factor associated with biological invasions. Aiming to contribute to the understanding of establishment of this relationship, the objectives of this study were to compare fruit phenology and to characterize the bird visitors of two palm trees, the native Euterpe edulis and the exotic Phoenix roebelenii in Alberto Löfgren State Park, southeastern Brazil. Phoenix roebelenii came from Southeast Asia and are often used in urban gardens. This palm is naturally disseminated in the forest understory of Alberto Löfgren State Park. However, currently, it is not considered an invasive species in Brazil. The phenological patterns of 30 adult individuals of each palm species were monitored monthly, between April 2012 and March 2014. Characterization of bird assemblages was obtained by focal observation, resulting in 10 samples of two hours per palm species. Palm trees differed in their phenological patterns showing partial overlap in phenology of ripe fruits, but not in their fruiting peak. The data obtained in this study suggest that the exotic palm tree has become an important food source for native birds of the Alberto Löfgren State Park and that due to this interaction seed dispersal occurs and probably contributes to the successful recruitment of this plant locally, which would justify the development of researches on the impact of its establishment on native plant species.
\end{abstract}

Keywords: birds; biological invasion; Atlantic Forest.

${ }^{1}$ Recebido para análise em 11.07.14. Aceito para publicação em 14.10.14

${ }^{2}$ Instituto Florestal, Rua do Horto, 931, 02377-000 São Paulo, SP, Brasil.

${ }^{3}$ Autor para correspondência: Alexsander Zamorano Antunes - alexza@if.sp.gov.br 


\section{INTRODUÇÃO}

Frutos de palmeiras (Arecaceae) são importante fonte energética para diversos vertebrados (Zona e Henderson, 1989; Zona, 2006). No Brasil, palmeiras nativas e exóticas são frequentemente incluídas no paisagismo, em parques públicos, praças, jardins e canteiros de avenidas (Lorenzi et al., 2004). Algumas das palmeiras exóticas apresentam potencial de invasão biológica, que pode prejudicar espécies de palmeiras ou de outras plantas nativas (Dislich et al., 2002; Mengardo et al., 2012).

Muitas das invasões biológicas estudadas iniciaram-se em áreas urbanas ou periurbanas (Rose, 1997). No caso das palmeiras, a dispersão de sementes por frugívoros nativos é um fator crucial para o sucesso do seu estabelecimento (Fonseca e Antunes, 2007; Mengardo et al., 2012). Assim, o objetivo geral do presente trabalho foi avaliar, em uma localidade periurbana, as assembleias de aves visitantes de duas palmeiras ornitocóricas sintópicas e que se dispersam naturalmente na área, a nativa juçara Euterpe edulis Mart. e a exótica tamareira-anã Phoenix roebelenii O'Brien.

Os objetivos específicos foram avaliar comparativamente, entre as duas espécies de palmeiras, a produção de diásporos e a disponibilidade de frutos maduros ao longo do ano. Também, se pretendeu caracterizar as assembleias visitantes considerando-se o comportamento de consumo dos frutos e o potencial de dispersão das sementes, com a finalidade de verificar diferenças entre as palmeiras nos padrões de interações com as aves. A partir da consecução desses objetivos, foram avaliadas as seguintes hipóteses:

1) as palmeiras não diferem em seus padrões fenológicos, apresentando alta sobreposição na disponibilidade de frutos maduros ao longo do ano;

2) considerando-se que as duas palmeiras produzem frutos de aparência similar, para consumidores visualmente orientados, existe alta similaridade na composição das assembleias de visitantes das duas palmeiras.

\section{MATERIAL E MÉTODOS}

O estudo foi desenvolvido na área administrativa do Instituto Florestal, no Parque Estadual Alberto Löfgren - PEAL, São Paulo-SP (2327'43'S, 46 37'59'W; $723 \mathrm{~m}$ de altitude; Figura 1). O parque ocupa uma área de 174 ha, com vegetação constituída por remanescente de Floresta Ombrófila Densa Montana em estádios inicial e médio de sucessão ecológica e por talhões reflorestados com espécies vegetais nativas e exóticas que, na maioria das vezes, apresentam um sub-bosque bem desenvolvido com predomínio de espécies vegetais nativas. O PEAL é contíguo ao Parque Estadual da Cantareira, que ocupa uma área de 7.900 ha. As duas Unidades de Conservação são consideradas, internacionalmente, uma "Important Bird Area - IBA", isto é, uma Área Importante para a Conservação das Aves (Bencke et al., 2006).

A juçara Euterpe edulis Mart. ocorre tanto nos remanescentes naturais quanto no sub-bosque dos talhões. Espécie nativa da Mata Atlântica e de matas de galeria do Cerrado, atinge até $20 \mathrm{~m}$ de altura, apresenta alta densidade em florestas não perturbadas e frutifica por um período superior a seis meses, produzindo grande quantidade de frutos que são consumidos por mais de 30 espécies de vertebrados (Galetti et al., 1999). Trata-se de espécie vulnerável à extinção no Brasil devido ao corte ilegal para a extração de palmito em toda a sua área de distribuição geográfica (Martinelli e Moraes, 2013).

Já a tamareira-anã Phoenix roebelenii O’Brien foi utilizada no paisagismo de áreas do PEAL, disseminando-se para alguns dos talhões (Figura 2). Originária do norte do Laos e do Vietnã e sudoeste da China, atinge de 2 a 4 metros de altura e é amplamente utilizada em projetos de paisagismo em todo o Brasil (Lorenzi et al., 2004; Iossi et al., 2007). Seus frutos são muito procurados por aves (Lorenzi et al., 2004; Fonseca e Antunes, 2007). 
OMOTE, T.; ANTUNES, A.Z.; MATSUKUMA, C.K. Fenologia e frugivoria de Euterpe edulis e Phoenix roebelenii

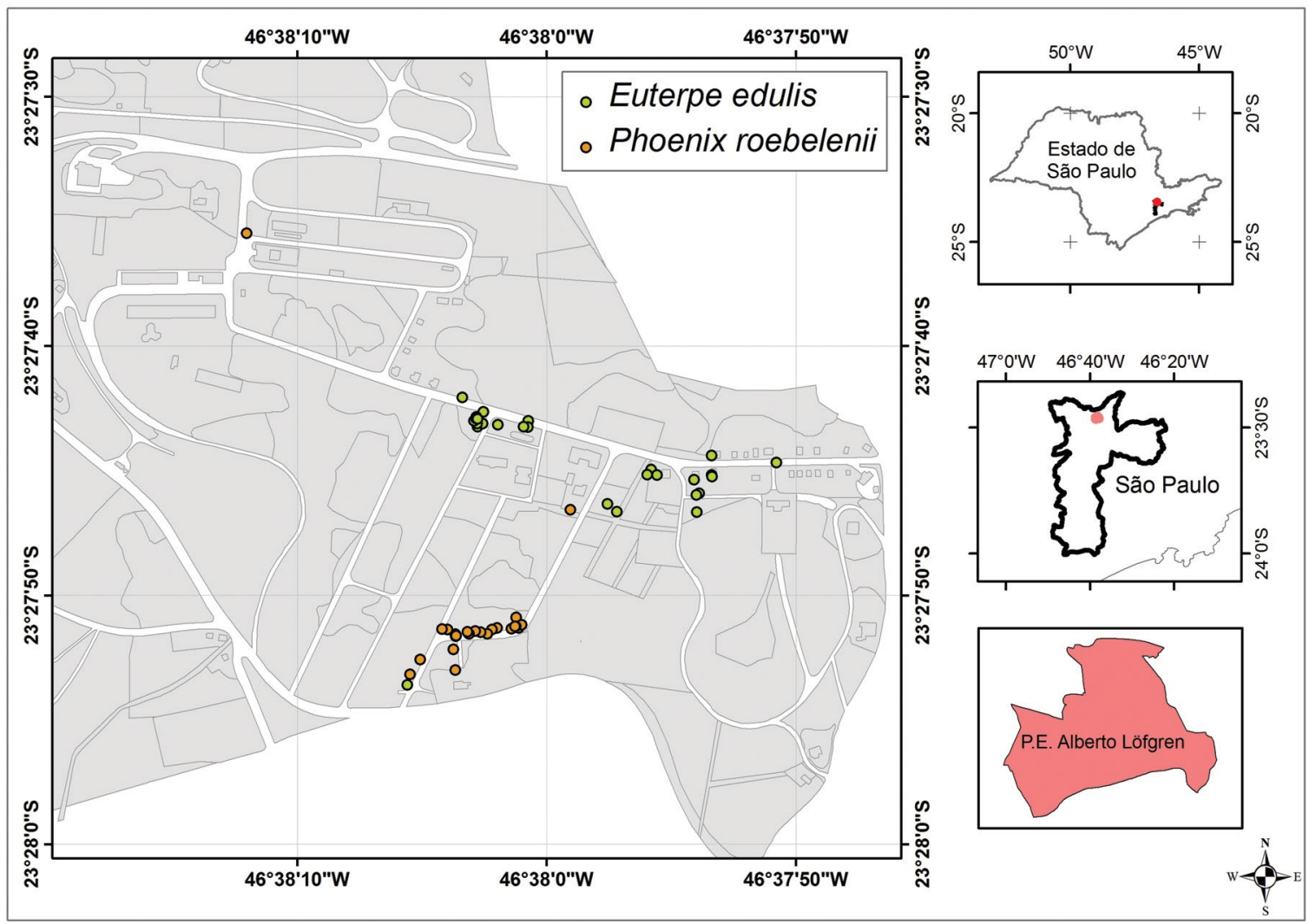

Figura 1. Localização do Parque Estadual Alberto Löfgren-SP, com a distribuição das palmeiras estudadas.

Figure 1. Location of Alberto Löfgren State Park, São Paulo state southeastern Brazil, with the distribution of sampled palm trees. 
OMOTE, T.; ANTUNES, A.Z.; MATSUKUMA, C.K. Fenologia e frugivoria de Euterpe edulis e Phoenix roebelenii

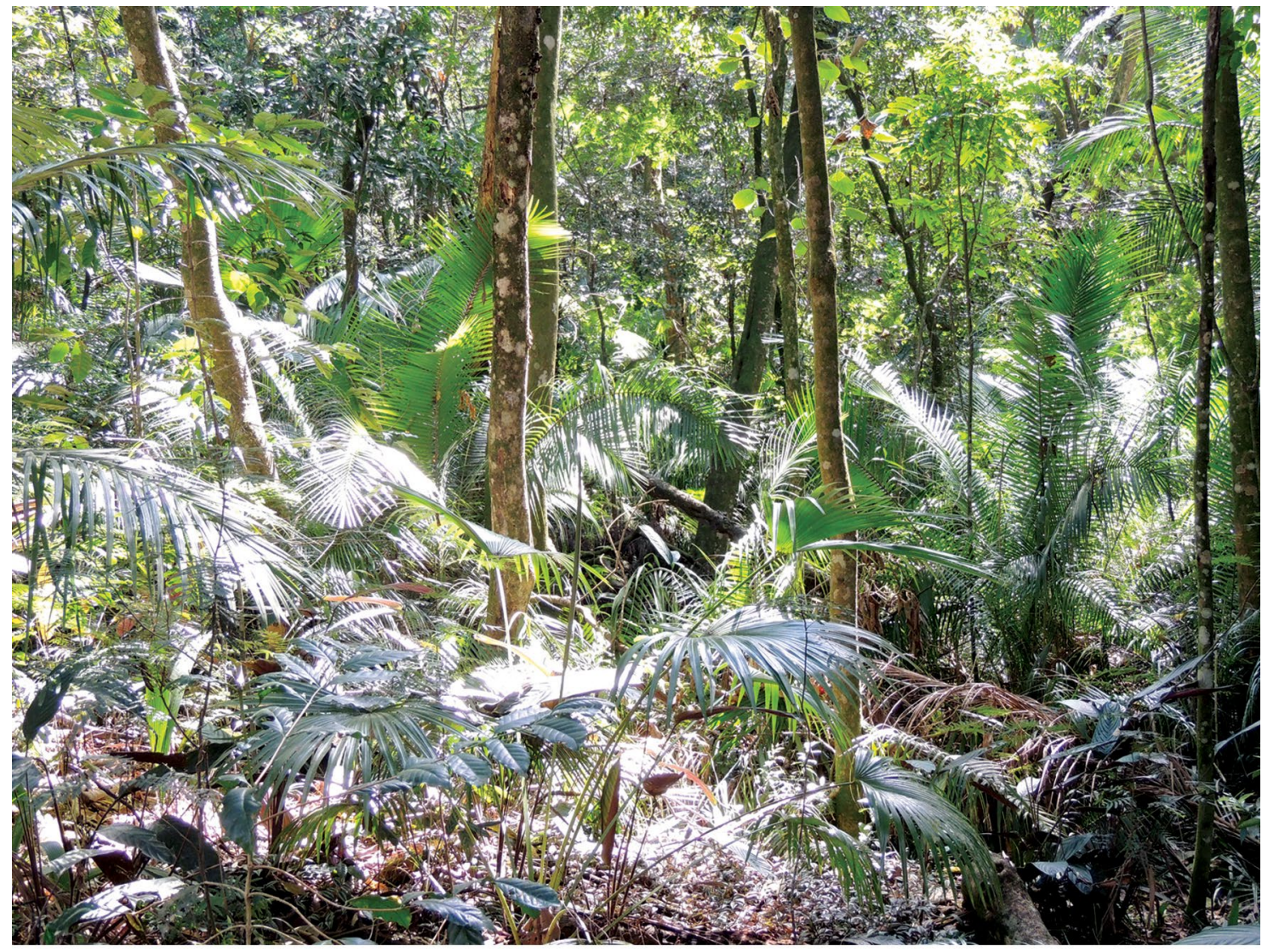

Figura 2. Detalhe de área do Parque Estadual Alberto Löfgren com presença da tamareira-anã Phoenix roebelenii no sub-bosque florestal.

Figura 2. Presence of Phoenix roebelenii at forest understory in Alberto Löfgren State Park. 
A coleta de dados ocorreu entre abril de 2012 e março de 2014. Inicialmente, foram percorridas as trilhas e estradas disponíveis, identificando-se as palmeiras adultas. Trinta indivíduos por espécie tiveram suas posições obtidas com GPS (Figura 1). Semanalmente, cada palmeira foi visitada registrando-se a fenofase em que esta se encontrava. No auge da fenofase frutos maduros, foi efetuada a observação focal (Lehner, 1979) para a caracterização da assembleia de aves visitantes. Cinco indivíduos de cada espécie de palmeira foram observados durante cinco dias em 2012 e cinco dias em 2013, por duas horas consecutivas diárias cada indivíduo, resultando em 10 amostras de visitação por espécie de palmeira. As observações ocorreram em maio de 2012 e de 2013 para Phoenix roebelenii e em setembro de 2012 e julho de 2013 para Euterpe edulis. Durante a fenofase frutos maduros do ano de 2012, foram coletados aleatoriamente 30 frutos por espécie de palmeira para a obtenção de medidas morfométricas. A média de frutos por espécie de palmeira foi obtida pela contagem de frutos de 10 cachos de 10 indivíduos de cada espécie. $\mathrm{O}$ número médio de frutos dos cachos foi multiplicado pelo total de cachos que apresentaram frutos maduros por espécie no período. A média obtida foi multiplicada pelo total de cachos que apresentaram frutos maduros no período.

As observações focais foram realizadas a uma distância aproximada de cinco metros das palmeiras, registrando-se as aves visitantes, bem como o seu comportamento, com auxílio de binóculos $8 \times 40$. As observações foram iniciadas em torno das 8h. O horário de coleta de dados foi definido a partir de um projeto-piloto, no qual se registrou o período de $8 \mathrm{~h}$ às $10 \mathrm{~h}$ como o de maior atividade das aves visitantes. Foi considerado um feeding bout cada vez que uma ave ou um grupo de aves foi observado consumindo um fruto independente da duração do comportamento alimentar (Galetti et al., 1999). As correlações da porcentagem de frutos maduros/mês entre as espécies de palmeira e da precipitação média mensal e o percentual médio de frutos maduros disponível por mês para cada espécie de palmeira foram obtidas por meio do coeficiente de postos de Spearman (rs). Valores médios são acompanhados por seu desvio-padrão e foram comparados por meio do teste Mann-Whitney (U). Os testes estatísticos foram efetuados com o programa BioEstat 5.0 (Ayres et al., 2008).
A similaridade na composição de espécies entre as assembleias de aves visitantes das duas palmeiras foi comparada por meio do índice de Jaccard - ISJ (Magurran, 1988).

Os dados de precipitação pluviométrica mensal foram obtidos no banco de dados do Instituto Nacional de Meteorologia e referem-se à estação do Mirante de Santana localizada a aproximadamente $4,5 \mathrm{~km}$ da área de estudo (Instituto Nacional de Meteorologia - INMET, 2014).

A nomenclatura utilizada para os táxons de aves segue a proposta do Comitê Brasileiro de Registros Ornitológicos (Comitê Brasileiro de Registros Ornitológicos - CBRO, 2014).

\section{RESULTADOS E DISCUSSÃO}

A produção média foi estimada em $8.669,5 \pm 2.073,84$ frutos por palmeira para Euterpe edulis e em $2.681 \pm 536$ para Phoenix roebelenii. $\mathrm{O}$ diâmetro médio dos frutos de E. edulis foi de $12,03 \pm 0,41 \mathrm{~mm}$ e de $9,93 \pm 0,74 \mathrm{~mm}$ para $P$. roebelenii, esta variável diferiu significativamente entre as duas palmeiras, $\mathrm{U}=7, \mathrm{p}<0,0001$.

Os picos de disponibilidade de frutos maduros diferiram entre as duas palmeiras nos dois anos de amostragem (Figura 3), resultando em correlação de baixa intensidade entre as porcentagens de frutos maduros entre as espécies, $r_{s}=0,18, p=0,39$. Essa fenofase variou acentuadamente sua distribuição mensal entre os anos considerados para E. edulis, mas não para P. roebelenii (Figura 3). Não houve correlação significativa entre a precipitação média mensal e o percentual médio de frutos maduros disponível por mês para esta última espécie, $r_{s}=-0,54, p=0,033$ (Figura 3). Já o percentual médio de frutos maduros de E. edulis apresentou correlação negativa significativa com a precipitação mensal, $r_{s}=-0,76, p<0,02$.

A variação no pico da fenofase frutos maduros entre anos para a palmeira juçara foi relatada em outras localidades do Sudeste do Brasil (Galetti et al., 1999). A estratégia fenológica de E. edulis, com maior produção de frutos, disponibilidade de frutos maduros a maior parte do ano e com variação nos picos dessa fenofase entre anos, poderia fazê-la ter mais sucessona ocupação dos talhões em relação a Phoenix roebelenii, fato que deve ser avaliado em pesquisas futuras. 


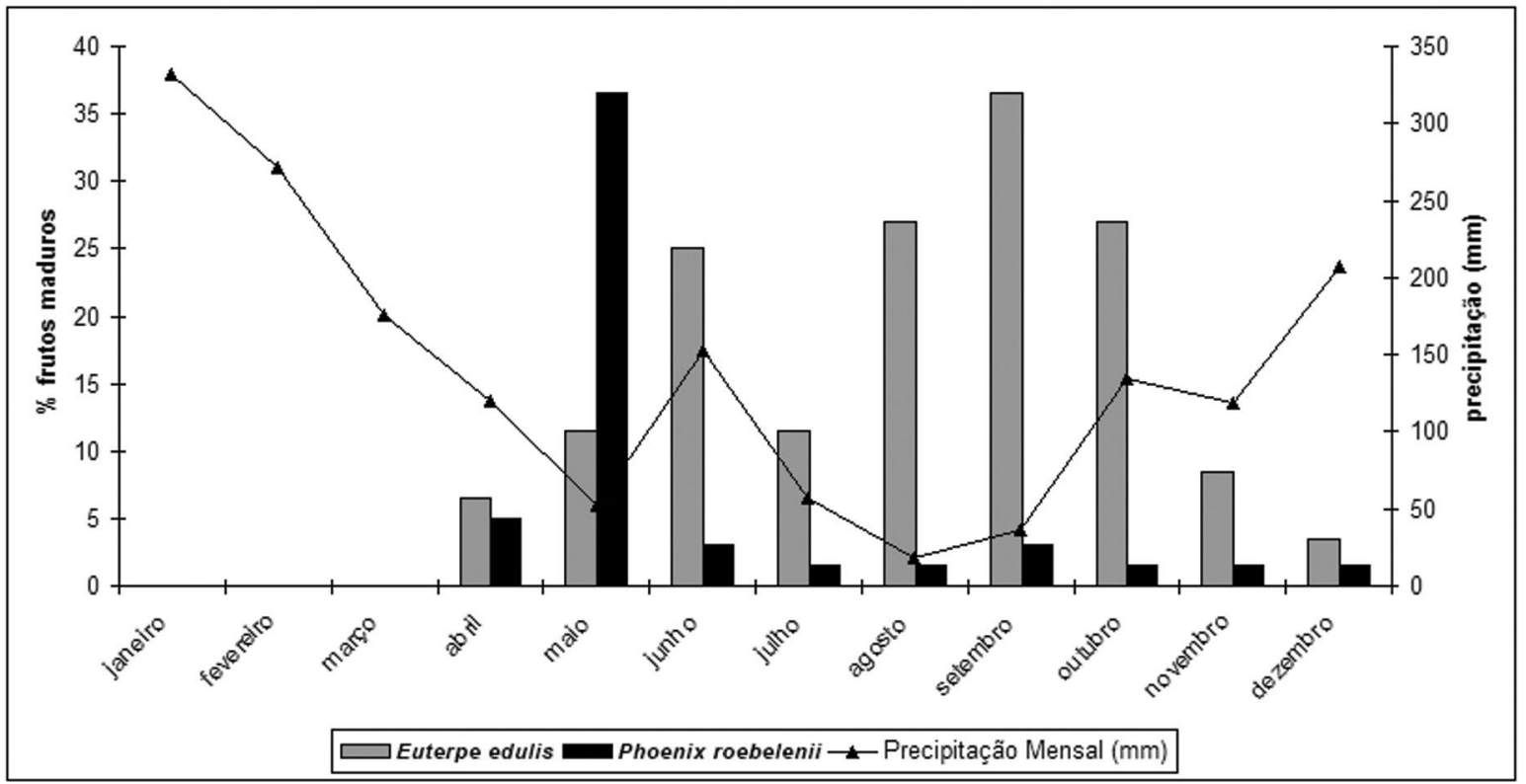

Figura 3. Distribuição percentual de frutos maduros das palmeiras Euterpe edulis e Phoenix roebelenii no período de abril de 2012 a março de 2014, em relação à precipitação média mensal, no Parque Estadual Alberto Löfgren-SP.

Figure 3. Monthly percentage distribution of ripen fruits of Euterpe edulis and Phoenix roebelenii palms, and average monthly precipitation in Alberto Löfgren State Park.

Quanto às aves visitantes, foram registradas 19 espécies para $P$. roebelenii e 10 para E. edulis (Tabela 1, figuras 4 e 5). Em ambas as palmeiras, a curva de acúmulo de espécies visitantes atingiu a assíntota após a sexta amostra (Figura 6). A assembleia de visitantes de $E$. edulis contou com uma espécie exclusiva, o pavó Pyroderus scutatus (Shaw, 1792). Esta espécie e a araponga Procnias nudicollis (Vieillot, 1817) são consideradas ameaçadas de extinção no Estado de São Paulo (São Paulo, 2014), sendo que a última também é considerada vulnerável internacionalmente (International Union for Conservation of Nature IUCN, 2014).
P. roebelenii apresentou riqueza média de visitantes por amostra, $8,9 \pm 1,45$, significativamente maior do que a obtida para $E$. edulis, $5 \pm 1,15, \mathrm{U}=0$, $\mathrm{p}<0,01$. A média de feeding bouts por amostra também diferiu significativamente entre ambas, $53,30 \pm 22,61$ e $21,20 \pm 12,41$, respectivamente, $\mathrm{U}=10,50, \mathrm{p}=0,0028$ (Tabela 2). Assim, o maior número de frutos produzidos por E. edulis e sua maior disponibilidade de frutos ao longo do ano em relação à $P$. roebelenii não se traduziram numa maior riqueza de espécies visitantes e maior número de feeding bouts. O tamanho menor dos frutos de $P$. roebelenii em relação aos de E. edulis pode ter atraído maior diversidade de aves visitantes, com distintos tamanhos corporais e aberturas de bico. 
OMOTE, T.; ANTUNES, A.Z.; MATSUKUMA, C.K. Fenologia e frugivoria de Euterpe edulis e Phoenix roebelenii

Tabela 1. Aves consumidoras dos frutos das palmeiras Euterpe edulis e Phoenix roebelenii no Parque Estadual Alberto Löfgren-SP, com o total de feeding bouts obtidos para cada espécie.

Table 1. Bird species consuming fruits of Euterpe edulis and Phoenix roebelenii palms in Alberto Löfgren State Park, with the feeding bouts.

\begin{tabular}{|c|c|c|c|}
\hline Nome do Táxon & Nome em Português & P. roebelenii & E. edulis \\
\hline \multicolumn{4}{|l|}{ Galliformes Linnaeus, 1758} \\
\hline \multicolumn{4}{|l|}{ Cracidae Rafinesque, 1815} \\
\hline Penelope obscura Temminck, 1815 & jacuaçu & 1 & 4 \\
\hline \multicolumn{4}{|l|}{ Piciformes Meyer \& Wolf, 1810} \\
\hline \multicolumn{4}{|l|}{ Ramphastidae Vigors, 1825} \\
\hline Ramphastos dicolorus Linnaeus, 1766 & tucano-de-bico-verde & 2 & 14 \\
\hline \multicolumn{4}{|l|}{ Psittaciformes Wagler, 1830} \\
\hline \multicolumn{4}{|l|}{ Psittacidae Rafinesque, 1815} \\
\hline Pyrrhura frontalis (Vieillot, 1817) & tiriba-de-testa-vermelha & 1 & 6 \\
\hline Brotogeris tirica (Gmelin, 1788) & periquito-rico & 1 & 0 \\
\hline \multicolumn{4}{|l|}{ Passeriformes Linnaeus, 1758} \\
\hline \multicolumn{4}{|l|}{ Cotingidae Bonaparte, 1849} \\
\hline Procnias nudicollis (Vieillot, 1817) & araponga & 2 & 24 \\
\hline Pyroderus scutatus (Shaw, 1792) & pavó & 0 & 3 \\
\hline \multicolumn{4}{|l|}{ Tyrannidae Vigors, 1825} \\
\hline Pitangus sulphuratus (Linnaeus, 1766) & bem-te-vi & 5 & 22 \\
\hline Megarynchus pitangua (Linnaeus, 1766) & neinei & 6 & 0 \\
\hline \multicolumn{4}{|l|}{ Turdidae Rafinesque, 1815} \\
\hline Turdus flavipes Vieillot, 1818 & sabiá-una & 50 & 74 \\
\hline Turdus leucomelas Vieillot, 1818 & sabiá-barranco & 78 & 37 \\
\hline Turdus rufiventris Vieillot, 1818 & sabiá-laranjeira & 220 & 23 \\
\hline Turdus amaurochalinus Cabanis, 1850 & sabiá-poca & 12 & 5 \\
\hline Turdus subalaris (Seebohm, 1887) & sabiá-ferreiro & 1 & 0 \\
\hline Turdus albicollis Vieillot, 1818 & sabiá-coleira & 13 & 0 \\
\hline \multicolumn{4}{|l|}{ Thraupidae Cabanis, 1847} \\
\hline Coereba flaveola (Linnaeus, 1758) & cambacica & 14 & 0 \\
\hline Tachyphonus coronatus (Vieillot, 1822) & tiê-preto & 1 & 0 \\
\hline Tangara sayaca (Linnaeus, 1766) & sanhaçu-cinzento & 66 & 0 \\
\hline Tangara palmarum (Wied, 1823) & sanhaçu-do-coqueiro & 47 & 0 \\
\hline Tangara cayana (Linnaeus, 1766) & saíra-amarela & 11 & 0 \\
\hline Dacnis cayana (Linnaeus, 1766) & saí-azul & 2 & 0 \\
\hline
\end{tabular}




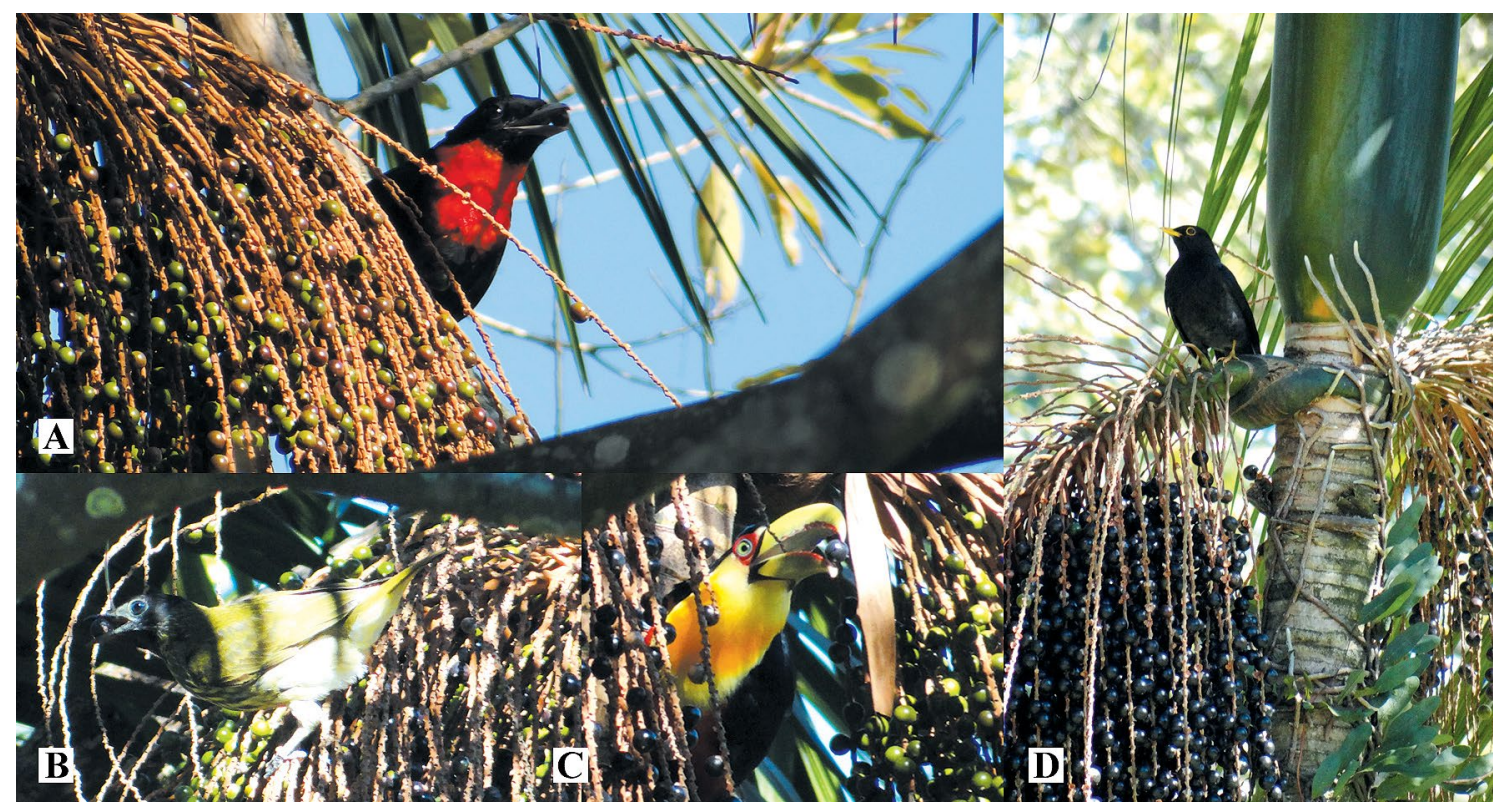

Figura 4. Aves consumidoras dos frutos da palmeira Euterpe edulis no Parque Estadual Alberto Löfgren-SP: Pyroderus scutatus (A), Procnias nudicollis (B), Ramphastos dicolorus (C) e Turdus flavipes (D).

Figure 4. Birds consuming fruits of Euterpe edulis palm in Alberto Löfgren State Park: Pyroderus scutatus (A), Procnias nudicollis (B), Ramphastos dicolorus (C) e Turdus flavipes (D).

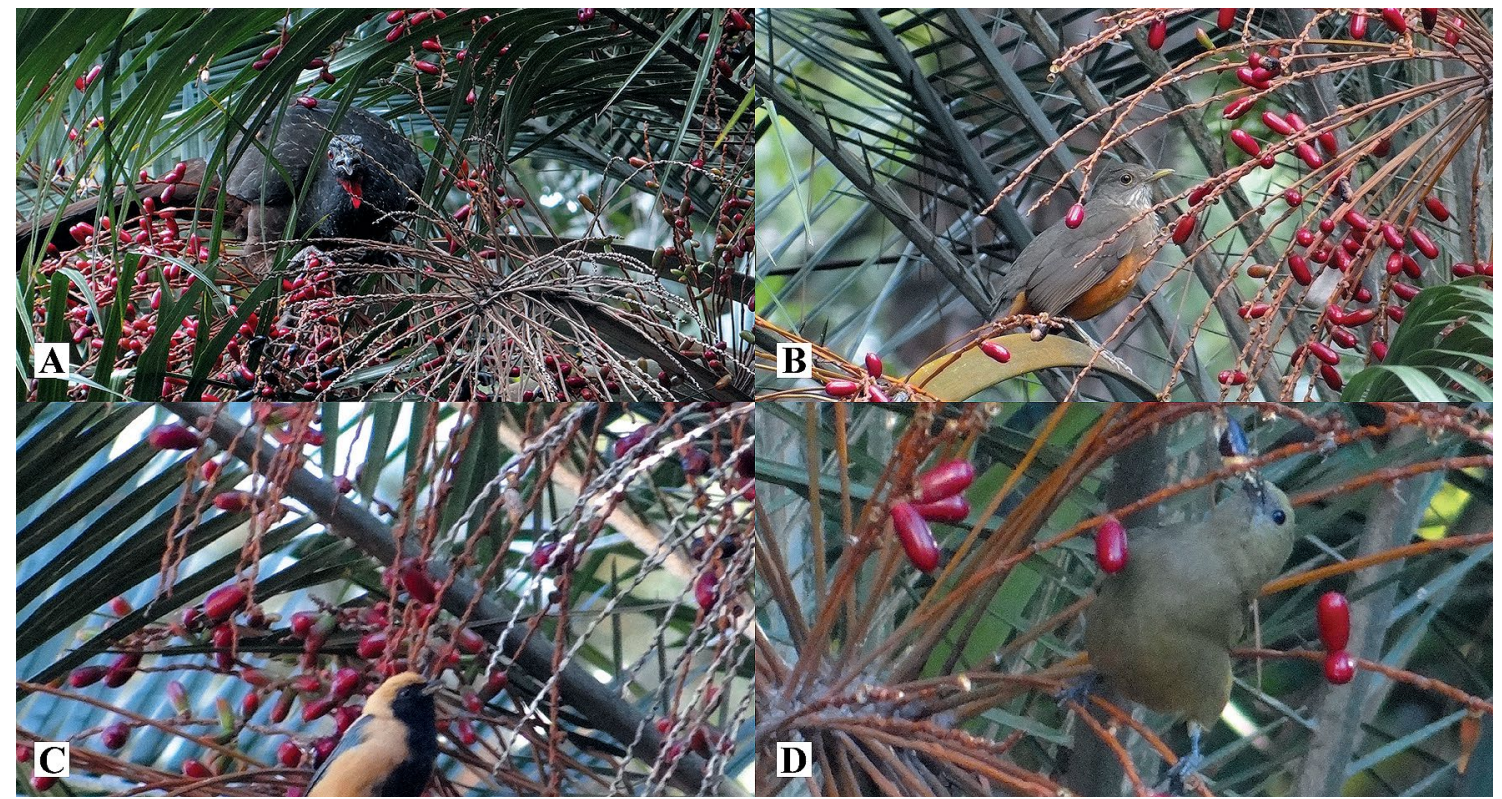

Figura 5. Aves consumidoras dos frutos das palmeiras Phoenix roebelenii no Parque Estadual Alberto Löfgren-SP: Penelope obscura (A), Turdus rufiventris (B), Tangara cayana (C) e Tangara palmarum (D).

Figure 5. Birds consuming fruits of Phoenix roebelenii palm in Alberto Löfgren State Park: Penelope obscura (A), Turdus rufiventris (B), Tangara cayana (C) e Tangara palmarum (D). 
OMOTE, T.; ANTUNES, A.Z.; MATSUKUMA, C.K. Fenologia e frugivoria de Euterpe edulis e Phoenix roebelenii

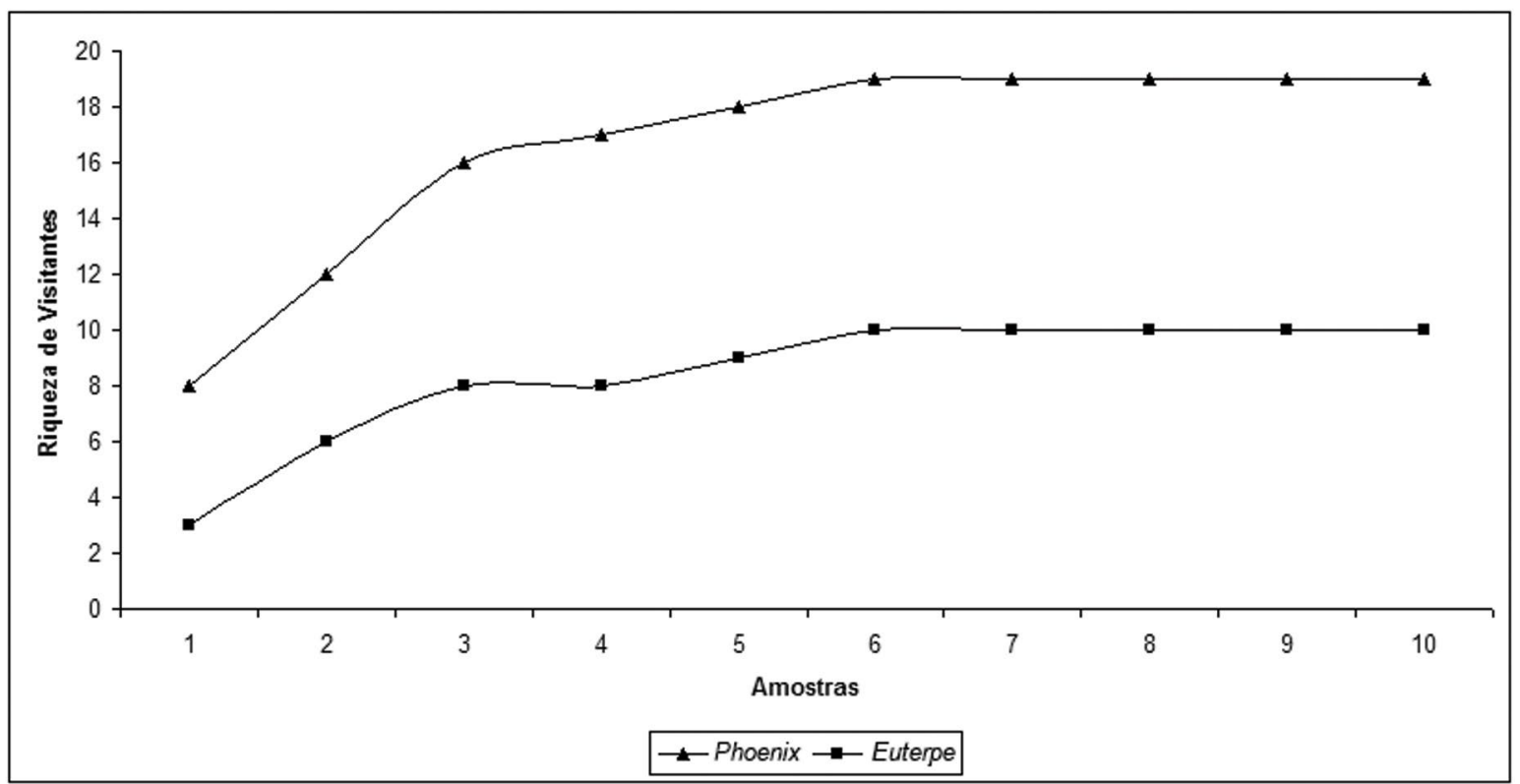

Figura 6. Curvas de acúmulo de espécies de aves visitantes das palmeiras Euterpe edulis e Phoenix roebelenii no Parque Estadual Alberto Löfgren-SP.

Figure 6. Species accumulation curves for bird visitor of Euterpe edulis and Phoenix roebelenii palms in Alberto Löfgren State Park.

Tabela 2. Total de feeding bouts por amostra, considerando-se todas as espécies de aves visitantes das palmeiras Euterpe edulis e Phoenix roebelenii no Parque Estadual Alberto Löfgren-SP.

Table 2. Total feeding bouts per sample, considering all species of birds visitors of palms Euterpe edulis and Phoenix roebelenii in Alberto Löfgren State Park.

\begin{tabular}{ccc}
\hline Amostra & \multicolumn{2}{c}{ Feeding bouts } \\
\cline { 2 - 3 } & Phoenix & Euterpe \\
\hline 1 & 23 & 6 \\
2 & 43 & 27 \\
3 & 68 & 40 \\
4 & 55 & 28 \\
5 & 47 & 15 \\
6 & 86 & 38 \\
7 & 82 & 16 \\
8 & 70 & 26 \\
9 & 32 & 9 \\
10 & 27 & 7 \\
\hline
\end{tabular}


A previsibilidade da frutificação de $P$. roebelenii em relação à de $E$. edulis também pode ter influenciado no fato de a primeira palmeira ter apresentado mais visitantes. A sobreposição parcial da fenofase frutos maduros, com picos em meses distintos para cada espécie, pode favorecer a dispersão das sementes de ambas as palmeiras. Em conjunto, elas ofertaram frutos maduros na maior parte do período de estudo. Isso pode ter contribuído para a manutenção de populações das aves frugívoras na área, principalmente dispersores de grande porte, que se deslocam por distâncias maiores, como o tucano-de-bico-verde Ramphastos dicolorus e a araponga Procnias nudicollis. Entretanto, cabe ressaltar que devido à variação fenológica de E. edulis, a sobreposição nos picos de frutificação entre as palmeiras variou entre os anos, indicando que pode haver maior competição por dispersores em determinados períodos.

A similaridade na composição de espécies entre as assembleias de aves visitantes das duas palmeiras foi moderada, ISJ $=0,45$. Restringindo-se a análise às espécies dispersoras potenciais de sementes, representantes das famílias Cracidae, Ramphastidae, Cotingidae, Tyrannidae e Turdidae, observa-se que $90 \%$ das visitantes de E. edulis e $58 \%$ das de $P$. roebelenii se enquadram nesta categoria, enquanto no caso dos feeding bouts, as dispersoras responderam por $97 \%$ e $73 \%$ do total de registros, respectivamente (Tabela 1). A similaridade na composição de dispersores potenciais entre as duas palmeiras foi maior do que a similaridade entre as assembleias de aves visitantes, ISJ $=0,66$.

Espécies do gênero Turdus foram as principais consumidoras dos frutos de ambas as palmeiras, contudo estas diferiram em relação à espécie dominante. Turdus rufiventris representou $41 \%$ dos registros de visitantes obtidos para P. roebelenii, enquanto Turdus flavipes constituiu $35 \%$ dos registros para E. edulis (Tabela 1). A observação do sabiá-ferreiro Turdus subalaris (Seebohm, 1887) consumindo fruto de P. roebelenii representa o primeiro registro desta ave para o Parque Estadual Alberto Löfgren (Antunes e Eston, 2008).

Os sabiás Turdus spp. são os principais dispersores de sementes de frutos ornitocóricos no PEAL (Fonseca e Antunes, 2007) e o sabiá-una é considerado o principal visitante da palmeira juçara $E$. edulis em várias localidades da Mata Atlântica (Galetti et. al., 1999; Cerisola et al., 2007, Fonseca e Antunes, 2007; Fadini et al., 2009; Castro et al., 2012). O comportamento dos sabiás pode fazê-los dispersores mais eficientes do que espécies de maior porte, pois eles tendem a permanecer por menos tempo nas palmeiras do que essas aves, provavelmente por enfrentarem um maior risco de predação (Howe, 1979; Silva et al., 2008). Tucanos e arapongas foram observados regurgitando sementes sob a palmeira visitada. Assim, faz-se necessário avaliar a efetividade da dispersão das sementes pelos dispersores potenciais (Schuppe, 1993; Galetti et al., 1999; 2000). Todavia, sabiás selecionam sementes menores de E. edulis, o que pode trazer consequência para o recrutamento e a dinâmica populacional desta palmeira em localidades em que dispersores de maior porte estejam ausentes, pois sementes pequenas apresentam maior mortalidade do que as maiores em condições de dessecação, e resultam em plântulas de menor porte (Galetti et al., 2013).

Psitacídeos atuaram como predadores de sementes, enquanto traupídeos retiraram pedaços da polpa dos frutos nos próprios cachos, algumas vezes descartando as sementes embaixo da palmeira-mãe.

Ambas as hipóteses testadas no estudo foram rejeitadas já que as palmeiras diferiram em seus padrões fenológicos apresentando sobreposição parcial na fenofase frutos maduros, mas não em seus picos de frutificação, e que devido ao menor número de espécies visitantes registradas para E. edulis, aproximadamente metade do total registrado para $P$. roebelenii, a similaridade na composição das assembleias de visitantes das duas palmeiras foi moderada. Cabe destacar que, considerando-se apenas os dispersores em potencial de ambas as espécies, apesar da maior similaridade neste subconjunto das assembleias de visitantes, observa-se que espécies de aves distintas foram responsáveis pelas maiores taxas de consumo de frutos obtidas para cada uma das palmeiras.

Os resultados indicam que a palmeira exótica $P$. roebelenii, tornou-se uma importante fonte de alimento para espécies de aves nativas no Parque Estadual Alberto Löfgren e que, em decorrência desta interação, ocorre a dispersão de suas sementes nesta Unidade de Conservação. 
No momento, a população invasora de $P$. roebelenii encontra-se estabelecida em áreas anteriormente alteradas pela ação humana, talhões de plantios de arbóreas não manejados. Entretanto, considerando-se que se trata de uma unidade de conservação de proteção integral, em cujo plano de manejo preconiza-se a restauração ecológica de suas áreas degradadas (São Paulo, 2012), pesquisas sobre o impacto do estabelecimento de $P$. roebelenii sobre as espécies vegetais nativas devem ser efetuadas.

A gestão de áreas protegidas precisa considerar as funções ecológicas das espécies, independente de sua origem geográfica, avaliando os benefícios ou prejuízos que estão produzindo à biodiversidade, saúde humana e serviços ecossistêmicos (Davis et al., 2011; Durigan et al., 2013). O monitoramento é fundamental, pois os efeitos de espécies exóticas ou nativas em desequilíbrio populacional podem variar ao longo do tempo, e as que não causam danos aos ecossistemas, no momento, podem vir a causar no futuro (Davis et al., 2011). O impacto ao ecossistema no início de um processo de invasão biológica é local, e nesta fase, a solução para o problema ainda é viável (McNeely et al., 2003). A tamareira-anã P. roebelenii ainda não é reconhecida como espécie exótica invasora no Brasil (Instituto Hórus, 2014), mas apresenta grande potencial de se dispersar pelo ambiente, pois, como demonstrado pelo presente estudo, várias espécies de aves se alimentam de seus frutos.

Em áreas em que espécies exóticas zoocóricas já estão estabelecidas e interagindo com a fauna nativa, ações de controle e erradicação devem considerar o impacto da diminuição de recursos alimentares para os animais frugívoros do local (Gosper et al., 2005; Mengardo e Pivello, 2012; Buckley e Han, 2014). Em ações de manejo futuras, que considerem a retirada ou controle da população de $P$. roebelenii no Parque, deve-se avaliar antes o impacto sobre a avifauna que consome seus frutos. Para isso, são necessárias pesquisas sobre a biologia dessas aves visando quantificar a importância dos frutos da tamareira-anã $P$. roebelenii em sua dieta e também avaliar as espécies vegetais nativas que apresentam frutos maduros no mesmo período do ano.

\section{AGRADECIMENTOS}

Agradecemos ao Pesquisador Geraldo Antonio Daher Corrêa Franco da Seção de Ecologia Florestal do Instituto Florestal pelo auxílio na identificação das palmeiras, e aos dois revisores anônimos pelas importantes críticas e sugestões.

\section{REFERÊNCIAS BIBLIOGRÁFICAS}

ANTUNES, A.Z.; ESTON, M.R. Avifauna do Parque Estadual Alberto Löfgren-São Paulo: diagnóstico e propostas para a conservação. Revista do Instituto Florestal, v. 20, n. 2, p. 195-211, 2008.

AYRES, M. et al. BioEstat 5.0: aplicações estatísticas nas áreas das ciências biológicas e médicas. Belém: Instituto de Desenvolvimento Sustentável Mamirauá, 2008. 364 p.

BENCKE, G.A. et al. (Org.). Áreas importantes para a conservação das aves no Brasil. Parte $\mathrm{I}$ Estados do Domínio da Mata Atlântica. São Paulo: SAVE Brasil, 2006. 494 p.

BUCKLEY, Y.M.; HAN, Y. Managing the side effects of invasion control. Science, v. 344, n. 6187, p. 975-976, 2014.

CASTRO, E.R. et al. Temporal variation in the abundance of two species of thrushes in relation to fruiting phenology in the Atlantic rainforest. Emu, v. 112, n. 2, p. 137-148, 2012.

CERISOLA, C.M.; ANTUNES, A.Z.; PORT-CARVALHO, M. Consumo de frutos de Euterpe edulis Martius (Arecaceae) por vertebrados no Parque Estadual Alberto Löfgren, São Paulo, Sudeste do Brasil. IF Série Registros, n. 31, p. 167-171, 2007.

COMITE BRASILEIRO DE REGISTROS ORNITOLÓGICOS - CBRO. Listas das aves do Brasil. 11. ed. versão 01/01/2014. Disponível em: $<$ http://www.cbro.org.br>. Acesso em: 6 ago. 2014.

DAVIS, M.A. et al. Don't judge species on their origins. Nature, v. 474, n. 7350, p. 153-154, 2011. 
DISLICH, R.; KISSER, N.; PIVELLO, V.R. A invasão de um fragmento florestal em São Paulo (SP) pela palmeira australiana Archontophoenix cunninghamiana H. Wendl. \& Drude. Revista Brasileira de Botânica, v. 25, n. 1, p. 55-64, 2002.

DURIGAN, G. et al. Control of invasive plants: ecological and socioeconomic criteria for the decision making process. Natureza \& Conservação, v. 11, n. 1, p. 23-30, 2013.

FADINI, R.F. et al. Effects of frugivore impoverishment and seed predators on the recruitment of a keystone palm. Acta Oecologica, v. 35, n. 2, p. 188-196, 2009.

FONSECA, F.Y.; ANTUNES, A.Z. Frugivoria e predação de sementes por aves no Parque Estadual Alberto Löfgren, São Paulo, SP. Revista do Instituto Florestal, v. 19, n. 2, p. 81-91, 2007.

GALETTI, M.; ZIPARRO, V.B.; MORELLATO, P.C. Fruiting phenology and frugivory on the palm Euterpe edulis in a lowland Atlantic Forest of Brazil. Ecotropica, v. 5, n. 2, p. 115-122, 1999.

.; LAPS, R.; PIZO, M.A. Frugivory by toucans (Ramphastidae) at two altitudes in the Atlantic Forest of Brazil. Biotropica, v. 32, n. 4b, p. 842-850, 2000.

et al. Functional extinction of birds drives rapid evolutionary changes in seed size. Science, v. 340, n. 6136, p. 1086-1090, 2013.

GOSPER, C.R.; STANSBURY, C.D.; VIVIAN-SMITH, G. Seed dispersal of fleshy-fruited invasive plants by birds: contributing factors and management options. Diversity and Distributions, v. 11, n. 6, p. $549-558,2005$.

HOWE, H.F. Fear and frugivory. American Naturalist, v. 114, n. 6, p. 925-931, 1979.

INSTITUTO HÓRUS. Base de dados nacional de espécies exóticas invasoras. Florianópolis I3N Brasil: Instituto Hórus de Desenvolvimento e Conservação Ambiental. Disponível em: <http:// i3n.institutohorus.org.br>. Acesso em: 10 jun. 2014.

INSTITUTO NACIONAL DE METEOROLOGIA INMET. BDMEP - Banco de Dados Meteorológicos para Ensino e Pesquisa. Disponível em: $<\mathrm{http}$ // www.inmet.gov.br/projetos/rede/pesquisa/inicio. php>. Acesso em: 16 abr. 2014.
INTERNATIONAL UNION FOR CONSERVATION OF NATURE - IUCN. IUCN red list of threatened species. Cambridge: IUCN Species Survival Commission. Disponível em: <http://www.redlist.org $>$. Acesso em: 26 set. 2014.

IOSSI, E. et al. Maturação fisiológica de sementes de Phoenix roebelenii O'Brien. Revista Brasileira de Sementes, v. 29, n. 1, p. 147-154, 2007

LEHNER, P. Handbook of ethological methods. New York: Garland STPM, 1979. 403 p.

LORENZI, H. et al. Palmeiras brasileiras e exóticas cultivadas. Nova Odessa: Plantarum, 2004. $416 \mathrm{p}$.

MAGURRAN, A. E. Ecological diversity and its measurement. Princeton: Princeton University Press, 1988. 179 p.

MARTINELLI, G.; MORAES, M.A. (Org.). Livro vermelho da flora do Brasil. Rio de Janeiro: Instituto de Pesquisas Jardim Botânico do Rio de Janeiro, 2013. 1100 p.

McNEELY, J.A.; NEVILLE, L.E.; REJMÁNEK, M. When is eradication a sound investment? Conservation in Practice, v. 4, n. 1, p. 30-31, 2003.

MENGARDO, A.L.; PIVELLO, V.R. Phenology and fruit traits of Archontophoenix cunninghamiana, an invasive palm tree in the Atlantic forest of Brazil. Ecotropica, v. 18, n. 1, p. 45-54, 2012.

et al. Comparing the establishment of an invasive and an endemic palm species in the Atlantic rainforest. Plant Ecology and Diversity, v. 5, n. 3, p. 345-354, 2012.

ROSE, S. Influence of suburban edges on invasion of Pittosporum undulatum into the bushland of northern Sydney, Australia. Australian Journal of Ecology, v. 22, n. 1, p. 89-99, 1997.

SÃO PAULO (ESTADO). Parque Estadual Alberto Löfgren: Plano de Manejo. São Paulo: Instituto Florestal, 2012. 710 p. Disponível em: <http:// s.ambiente.sp.gov.br/if/Plano_de_Manejo_PE_ Alberto_Lofgren_pdf $>$. Acesso em: 10 jun. 2014. 
SÃO PAULO (Estado). Decreto $\mathrm{n}^{0}$ 60.133, de 7 de fevereiro de 2014. Declara as espécies da fauna silvestre ameaçadas, as quase ameaçadas e as deficientes de dados para avaliação no Estado de São Paulo e dá providências correlatas. Diário Oficial do Estado de São Paulo, Poder Executivo, v. 124, n. 27, 8 fev. 2014. Seção I, p. 25-32, 2014.

SCHUPPE, W. Quantity, quality, and the effectiveness of seed dispersal by animals. Vegetatio, v. 107/108, n. 1, p. 15-29, 1993.

SILVA, I.A.; FIGUEIREDO, R.A.; MATOS, D.M.S. Feeding visit time of fruit-eating birds in Cerrado plants: revisiting the predation risk model. Revista Brasileira de Zoologia, v. 25, n. 4, p. 682-688, 2008.

ZONA, S. Additions to "a review of animal-mediated seed dispersal of palms". 2006. Disponível em: $<$ http//:www.virtualherbarium.org/palms/psdispersal.html $>$. Acesso em: 16 maio 2014.

ZONA, S.; HENDERSON, A. A review of animal-mediated seed dispersal of palms. Selbyana, v. 11, p. 6-21, 1989. 